\title{
Performance Optimization in WBAN Using Hybrid BDT and SVM Classifier
}

\author{
Madhumita Kathuria \\ YMCA University of Science and Technology, Faridabad, India \\ E-mail: madhumita.fet@mriu.edu.in \\ Sapna Gambhir \\ YMCA University of Science and Technology, Faridabad, India \\ E-mail: sapnagambhir@gmail.com
}

\begin{abstract}
Wireless Body Area Network has attracted significant research interest in various applications due to its self-automaton and advanced sensor technology. The most severe is sue in WBAN is to sustain its Quality of Service (QoS) under the dynamic changing environment like healthcare, and patient monitoring system. Another critical is sue in WBAN is heterogeneous packet handling in such resource-constrained network. In this paper, a new classifier having hybrid Binary Decision Tree and Support Vector Machine classifier is proposed to tackle these important challenges. The proposed hybrid classifier decomposes the $\mathrm{N}$-class classification problem into N-1 sub-problems, each separating a pair of subclasses. This protocol dynamically updates the priority of packet and node, adjusts data rate, packet transmission order and time, and resource distribution for the nodes based on node priority. The proposed protocol is implemented and simulated using NS-2 network simulator. The result generated for proposed approach shows that new protocol can outperform in a dynamic environment, and yields better performance by leveraging advantages of both the Binary Decision Tree in terms of efficient computation and Support Vector Machine for high classification accuracy. This hybrid classifier significantly reduces loss ratio and delay and increase packet delivery ratio and throughput.
\end{abstract}

Index Terms - Binary decision tree, Multi-class packet classification, Packet prioritization, Support vector machine, Wireless Body Area Network.

\section{INTRODUCTION}

A Wireless Body Area Network (WBAN) consists of a number of tiny sensors located on or in the human body, depending on the needs of a patient. These sensors are equipped with a wireless interface and are capable of sensing the required health data and can transmit the data to a central Controller Unit (CU), such as a personal digital assistant (PDA) or a smart phone, for preprocessing. The goal of the healthcare WBAN architecture is to remotely sense heterogeneous vital signals and to provide an alert message in critical conditions. This system will reduce the need of dedicated medical personnel services for patient's health status monitoring and help the patients to lead a normal life besides providing them with the high quality of medical services. It is used for storing the health-related data, to analyze the progress of health condition over a period of time, to predict an emergency situation, and to sending an alert message to the Medical Server Unit (MSU). The pre-processed data can be used by the medical personnel for further clinical diagnosis.

For provid ing better Quality of Service (QoS) [1-10] in the field of a heterogeneous WBAN, accurate classification of traffic is much essential. The proposed protocol has the ability to classify data packets and assign them different priorities guaranteeing a certain level of performance in terms of better data delivery, reduced delay and improved throughput [11]. It has the capability to update the various parameters dynamically and adjusts them accordingly.

The main goal of the proposed architecture is to design an approach for packet classification to enhanced resource utilization and improve the quality of medical services. In this paper, an advanced step in the direction of designing such an efficient clas sification [12-14] algorith $m$ was taken. The proposed packet classification unit tries to categorize packets into different classes and assigns each of them a priority. For doing the same, the classification unit needs to have a multi-class classification technique. The Support Vector Machine (SVM) classifiers [15-19] are multi-class classifications which often have superior recognition rates in comparis on to other classification methods. In SVM, the multi-class classification problems usually decompose into several two-class problems using several Binary Decision Trees (BDTs) [20-25]. So we optimize the performance of the packet classification unit with the help of proposed hybrid Binary Decision Tree and Support Vector Machine based classifier, which utilizes a binary tree based architecture that further utilizes SVMs for solving multi-class problems [25-29]. The proposed classifier leverages advantages of both the Binary Decision Tree in terms of efficient computation and SVMs for high classification accuracy. In this paper, we empirically investigate the performance of hybrid classifier and find that this hybrid approach yields 
noticeable better analytical performance.

The organization of the paper is as follows: Section II presents the work done so far on this aspect. Section III illustrates the proposed protocol. Section IV describes the proposed hybrid classifier algorith $\mathrm{m}$. Section V compares the performance analysis of the proposed protocol along with existing protocol. Section VI concludes the paper.

\section{RELATED WORK}

The existing work related to traffic flow classification is given below.

The architecture in Optimized Congestion Management Protocol (OCMP) for Healthcare W ireless Sensor Networks [10] assigns dynamic weights to each child node and follows a fair queue management policy. If any child node's queue is likely to be full, then the free space of any other child node's queue can be utilized. This approach helps in minimizing the packet drop and loss rates for high-priority traffic, reducing congestion, and providing fair scheduling. It also utilizes bandwidth in a better way and achieves better throughputs; however this approach deficient in detection and classification of heterogeneous traffic flows in a frequently changing and dynamic environment of a healthcare system.

In paper [12], authors describe the marg in line between hard and soft classification techniques. According to them, soft classifier explicitly estimate the probabilities and then perform classification based on estimated probabilities. In contrast, hard classifier directly target on the classification decision boundary without producing the probability estimation.

Authors provide a study and analys is of various packet classification techniques in [13-14]. They have explored their ideas in this field and tried to briefly highlights advantages, disadvantages, usage and the platforms of their techniques.

In paper [26], a novel architecture of Support Vector Machine classifiers utilizes binary decision tree (SVMBDT) for solving multi-class problems has been presented. The clustering algorithm that utilizes Euclidean distance measures at the kernel space is used to convert the multi-class problem into a binary decision tree. Euclidean distance is used to measure the similarities between classes. The results of the experiments show that the speed of training and testing are improved while keeping comparable or offering even better than in the other SVM multi-class methods.

Classifiers proposed in [27-29] describe the use of SVM-BDT methods. The study proves that the testing time of SVM-BDT is noticeably better than the oneagainst-all and one-against-one methods. The SVM-BDT method was faster in the recognition phase for the Pendigit dataset, but slightly slower than DAGSVM method for the Statlog dataset.

All the limitations of existing protocol and the requirements of multi-class problem for multi-objective heterogeneous traffic classification motivate us to design a noble protocol which effectively improves the speed and comprehensive performance of the healthcare
WBAN system.

\section{PROPOSED ARCHITECTURE}

Nowadays, the monitoring of patient health conditions is becoming more important for immediate and emergency medical services. Furthermore, many vital signals are required to be monitored continuously, and transmitted within the time bound. The proposed architecture as discussed in [30] having a Controller Unit (CU), which gathers data packets from different sensor nodes, and forwards them in a definite order towards the medical server unit (MSU), positioned in a remote place. The order of packet forwarding depends on its priority, and the priority is obtained after concerning classification policies. Classification of packets with guaranteed Quality of Service (QoS) in a heterogeneous and dynamic environment is a challenging task. In this paper, we have studied the existing protocols and identified all issues related to classification, emergency services, and resource utilization in a frequently changing environment of WBAN. These limitations in existing protocols help us to introduce a new dignified hybrid classificat ion technique in the proposed protocol, and the simulation results show that the proposed protocol outperforms existing ones in terms of throughput, delay and packet delivery ratio.

\section{A. Wireless Body Area Network (WBAN)}

All the sensors equipped in WBANU are capable of sensing the required data and transmit the data to a central Controller Unit (CU).

In Data sensing and Pre-processing unit, the vital signal is sensed and processed into the desired format. This unit also calculates the data sending rate, packet transmission gap, and bandwidth allocation for each sensor node dynamically, where the data sending rate is defined as the number of packets to be sent in a given interval. Packet Transmission time gap is described as the transmission time gap between two consecutive packets, and the bandwidth allocation is defined as the amount of bandwidth assigned to individual nodes.

The Packet Dispatching unit performs classification, queuing and scheduling of packet at sensor node according to their traffic or flow type. The real-time bandwidth deficient traffic flows are assigned a higher priority than the non-real-time traffics. Hence, they are queued into the high priority queue and delivered to the CU with minimum possible delay. Its main motive is to handle heterogeneous packet-flow while reducing delay and starvation.

\section{B. Controller Unit (CU)}

The Controller Unit aggregates all received data from various sensor nodes in the Aggregation unit, and updates database accordingly. It also pre-processes packets and transmits them to medical server unit for diagnosing purposes.

The Packet handling unit maintains both the data and control packets in the heterogeneous WBANs. This unit is also responsible for the detection of critical situations; 
classification, queuing and scheduling of packets; and updating of priorities in a dynamic healthcare environment.

The main responsibility of the Alerting unit is immediate detection and notification of critical or emergency conditions. Due to uncertainty condition of patient, accurate detection of the condition is a necessary requirement for the healthcare system. Fewer and uneven observations give an imperfect depiction of what is actually happening, thus resulting in an inaccurate diagnosis. So this unit tries to detect the critical condition of a patient by focusing on multiple discrete observations for a particular time interval and not just by observing a single observation, this gives a better picture of what is happening and remove inaccurate results.

Upon reception of a packet, it checks for the variation in sensed values. If it identifies variation in sensed values, then it calculates their standard deviation. If the standard deviation value is greater than the critical threshold value (i.e. provided by the medical personal), then it activates the alert index field in the packet header and transmits it to classification unit.

The main job of Packet classification unit is the classification of packets based on the traffic or flow and level of criticality, and assigning them a unique priority. It categorizes the heterogeneous traffic into one of these categories: Real-time traffic, Alert or Emergency traffic, On_Demand traffic, and Normal traffic. Real-time packets are stored and sent when an appropriate amount of bandwidth is available. The Alert packet has the highest priority and is sent immediately without any delay. On_Demand packets are sensed and sent to doctors on their request. Normal packets are sent in a routine way.

The Queuing unit holds packets until the scheduler fetches and serves them. The proposed protocol uses two Double-Ended Priority Queues (DEPQs). One is used to store high priority packets i.e. Alert or Real-time packets and another is used to store low priority packets i.e. On_Demand or Normal packets.

The proposed Scheduling unit presents a new scheduling approach, called Rate based Earliest Deadline First (REDF) scheduling, which is used to reduce the queuing time and drop rates. The REDF finds and drops those low-priority packets whose deadline is exceeded. The main idea behind this scheduling is resource utilization. The strength of this method is that it deals with heterogeneous flows with considerably different bandwidth requirements. It also avoids the starvation and delay problem, faced by low-priority queues and low priority packets.

In the Prioritization unit, the priority index of the sensor changes dynamically over the time by activating prioritization field. The priority is updated by the medical person by analyzing some fields of packet i.e. sensor node's previous priority and level of criticality. A medical person can also ask for some On-Demand data by making this field active.

\section{Medical Server Unit (MSU)}

MSU receives data from the $\mathrm{CU}$ and takes decisions intelligently.

In Packet monitoring unit, MSU collects information about vital signals of a patient, displays them on the screen and stores them for later processing. A medical database is built on the workstation computer, which stores the patient's identification data, physiological data and otherdiagnosis related data.

The Decision making unit provides a filter facility. If the received packet is the highest priority packet with alert index $=1$, then MSU issues an alert message to the medical personal. Upon reception of an alert message, the medical person might request On_Demand data from the patient or can update old values of some pre-defined parameters i.e. priority of sensor node, vital signal range, monitoring time etc.

\section{PRoposed PACKet Classifier}

During classification, packets are categorized into distinct data traffics and then served accordingly, so Packet classification is an essential processing task in CU. It is a mechanism that categorizes packets into an appropriate class and assigns them a specific priority. Some attributes of a packet header, i.e. packet flow type, packet size, bandwidth, On_Demand index and Alert index fields are mapped into priority index. The priority is used to determine the order in which packets have to be served or transmitted to the MSU during a particular time period. The Packet classification unit classifies the entire traffic into four categories; Normal, On-demand, Realtime, and Alert or Emergency traffic. Normal traffic is transmitted in an uninterrupted manner. This includes unobtrusive and routine health monitoring of a patient. On-Demand traffic is initiated by the medical person to know certain information, mostly for the purpose of diagnosis and prescription. Real-time traffics are transmitted as soon as they are generated if bandwidth is available. Alert traffic is commenced when sensed value exceeds a predefined threshold range and should be transmitted within an acceptable delay. The alert traffic is not generated on a regular basis and is totally unpredictable.

The proposed Packet classification unit works by applying novel classifier employing the hybrid approach of both Binary Decision Tree and Support Vector Machine (i.e. named as BDSVMT) on multiple header fields of a packet. This classifier compares header fields of incoming packets with the set of predefined set of rules and decides their priority.

A Binary Decision Tree (BDT) is a rule-based classifier technique, where the rules are normally expressed in the form if-then statements. These rules are then converted into a binary decision tree while a Support Vector Machine (SVM) is based on statistical learning theory, classifies data by determining a set of support vectors that are members of the set of training inputs that outline a hyperplane in feature space.

This hybrid classifier is very much useful for multiclass packet classifications, which usually decompose the multi-class categories into several two-class groups by 
utilizing binary decision tree. This classifier provides a tree-based architecture as shown in Fig. 1, contains binary SVM in the internal nodes.

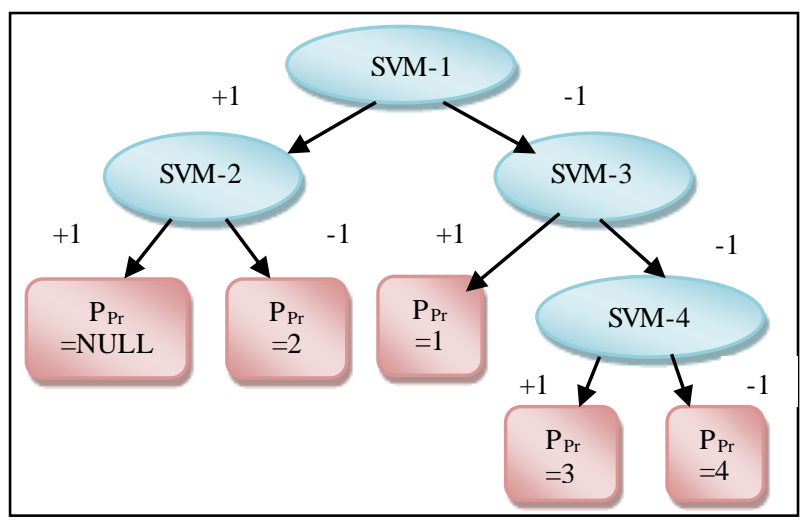

Fig.1. The Hybrid Packet classifier.

In this classifier; N-1 SVMs are needed to be trained for an $\mathrm{N}$ class problem. The proposed classification unit consists of five classes related to heterogeneous traffic, so needs four SVMs. These traffics are organized into two groups, since SVM at each node of BDT divides into two outputs $(+1$ for the positive group and -1 for the negative group). It then assigns each class with a particular priority index (i.e. 1 for Alert traffic, 2 for bandwidth deficient Real-time traffic, 3 for On_Demand traffic, 4 for Normal traffic, and NULL for forwarded Real-time traffic).

The rule set consists of five rules to provide five class of prio rity, as given in Table 1 , where $R=\left\{R_{1}, R_{2}, R_{3}, R_{4}\right.$, $\left.\mathrm{R}_{5}\right\}$ denotes the set of rules. RT denotes the Real-time traffic or flow, NRT denotes the Non-Real-time traffic or flow, $\mathrm{P}_{\text {Size }}$ denotes the size of the packet, $\mathrm{A}_{\text {Index }}$ denotes the value of the alert index field, $\mathrm{BW}_{\mathrm{av}}$ denotes the availability of Bandwidth, $O D_{\text {Index }}$ denotes the value of the On_Demand index field.

Table 1. Rule set table for packet classification

\begin{tabular}{|c|c|c|c|c|c|c|}
\hline Rule & $\begin{array}{c}\text { RT/ } \\
\text { NRT }\end{array}$ & P $_{\text {Size }}$ & BW $_{\text {av }}$ & A Index $_{\text {In }}$ & OD $_{\text {Index }}$ & Priority \\
\hline$R_{1}$ & RT & fixed & Yes & - & - & Null \\
\hline$R_{2}$ & RT & fixed & No & - & - & 2 \\
\hline$R_{3}$ & NRT & fixed & - & 1 & 0 & 1 \\
\hline$R_{4}$ & NRT & fixed & - & 0 & 1 & 3 \\
\hline$R_{5}$ & NRT & fixed & - & 0 & 0 & 4 \\
\hline
\end{tabular}

The Intermediate node of each Binary Decision based SVM tree (BDSVMT) follows the above specific rules and assigns the priority accordingly.

\section{A. Mathematical Formulation}

The aim of the hybrid classifier is to create a statistical model using SVM in BDT to predict the priority value ( $p r)$ of a packet $P$ by considering the packet header attribute as vector $\mathrm{v}_{\mathrm{i}}$.

Assume a set of packet field samples say $P^{d}$ taken from a packet, and represented as:

$$
\left\{v_{i} \mid p r_{i}\right\}_{i=1}^{d}
$$

where $v_{i}$ is the value of attribute $i$ in the header field of the packet, $\mathrm{pr}_{\mathrm{i}}$ is the priority inde $\mathrm{x}$ and $\operatorname{pr}_{\mathrm{i}} \in\{1,2,3,4$, and NULL\}.

The separating hyper-plane is defined using Eq. (2).

$$
f\left(v_{i}\right)=w * \emptyset\left(v_{i}\right)+b
$$

where $w$ is the weight, $b$ is the optimal bias, and $\phi$ is the nonlinear mapping function applied to input vectors and is expressed as:

$$
\begin{gathered}
\emptyset(v)=\left\{\begin{array}{l}
\left(P^{R T} \mid \text { Flow } \text { type }=R T\right) \\
\left(P^{N R T} \mid \begin{array}{r}
\text { otherwise })
\end{array}\right.
\end{array}\right. \\
\text { where } P^{R T}=\left\{\begin{array}{l}
\left(p r=2 \mid \text { Psize }>B W_{a v}\right) \\
(p r=\text { Null } \mid \text { otherwise })
\end{array}\right. \\
P^{N R T}=\left\{P^{\left.A_{\text {index }} \mid P^{O D_{\text {index }}}\right\}}\right.
\end{gathered}
$$

where $P^{R T}$ denotes the Real-time field, $P^{N R T}$ denotes the Non-real-time field, $P_{\text {Size }}$ denotes packet size field, $P^{A_{\text {index }}}$ denotes the Alert index field $P^{O D_{\text {index }}}$ denotes the On_Demand index field, and $p r$ denotes priority field.

The optimization is done by minimizing $w$ which results in maximized distance between the closest point of the hyper-plane and the hyper-plane itself. It can be understood as:

$$
\min (\varnothing(w))=\frac{1}{2} *\|w\|^{2}+c \sum_{i=1}^{P^{d}} e_{i}
$$

where $c$ is the constant used for regularization, and $e_{i}$ is the normalized variation with $e_{i}>=0$.

The output function can be expressed as:

$$
O\left(w * \varnothing\left(v_{i}\right)+b\right) \geq 1-e_{i}
$$

where $i=1 \ldots P^{d}$.

Applying Lagrangian method

$\max L_{1}(a)=\sum_{i=1} P^{d}-\frac{1}{2} \sum_{i, j=1}{ }^{d} a_{i} a_{j} o_{i} o_{j}\left(\emptyset\left(v_{i}\right) * \varnothing\left(v_{j}\right)\right)$

such that

$$
\sum_{i=1}^{P^{d}} a_{i} o_{i}=0
$$

where $a$ is the Lagrangian multiplier, and

$$
c \geq a_{i} \geq 0
$$


On solving the above equation, the classification can be expressed as given below:

$$
L_{e}= \begin{cases}0, & \text { if }\left|o_{i}-f\left(v_{i}\right)\right| \leq e \\ \left|o_{i}-f\left(v_{i}\right)-e\right|, & \text { if }\left|o_{i}-f\left(v_{i}\right)\right|>e\end{cases}
$$

where $e$ is the maximum allowed error.

In the training set, all the above five ru les with multiple attributes are used to define the priority, while the test set is used validate it in simulation and to determine the accuracy of this classifier in term of performance.

The proposed classifier yields faster search and recognition speed, which improves the performance, is significantly better than other protocols. The main focus of proposed hybrid classifier is to determine the optimized solution by applying SVM functions on binary decision rules, where BDTs are much faster than SVMs in classifying new instances while SVMs perform better than BDTs in terms of classification accuracy.

\section{PERFORMANCE ANALYSIS}

In this section, the simulation process is done to evaluate the performance of the proposed protocol under different scenarios. The simulation measures the performance of the proposed protocol (denoted as $\mathrm{pr}_{-}$) with the performance of the existing OCMP protocol (denoted as ex_). The implementation is done using the network simu lator NS-2.35. The performance metrics that reflect the most fundamental characteristics of proposed protocol can be categorized into three main facets: in terms of parameters like Packet Delivery Ratio, Throughput, and End-to-End Delay with respect to variation in number of nodes. The results of these three metrics are shown using Xgraphs (i.e. an analysis tool for NS-2.35)

\section{A. Packet Delivery Ratio(PDR)}

The PDR is defined as the ratio of a total number of packets received by the receiver to the total number of packets transmitted from the source node. PDR can be calculated using Eq. (13).

$$
P D R=\frac{\sum_{k=1}^{n} P_{k}^{\text {Transmit }}-\sum_{k=1}^{n} P_{k}^{\text {Lost }}}{\sum_{k=1}^{n} P_{k}^{\text {Transmit }}} * 100
$$

where $P^{\text {Lost }}$ denotes the number of packets lost in the transmission and $P^{\text {Transmit }}$ denotes the number of packets transmitted from the source node.

The comparison graph for PDR is given in Fig. 2, shows that proposed protocol succeeds to transmit and receive a large number of packets than the existing one because more amount of and more useful packets are transmitted in the preliminary phase.

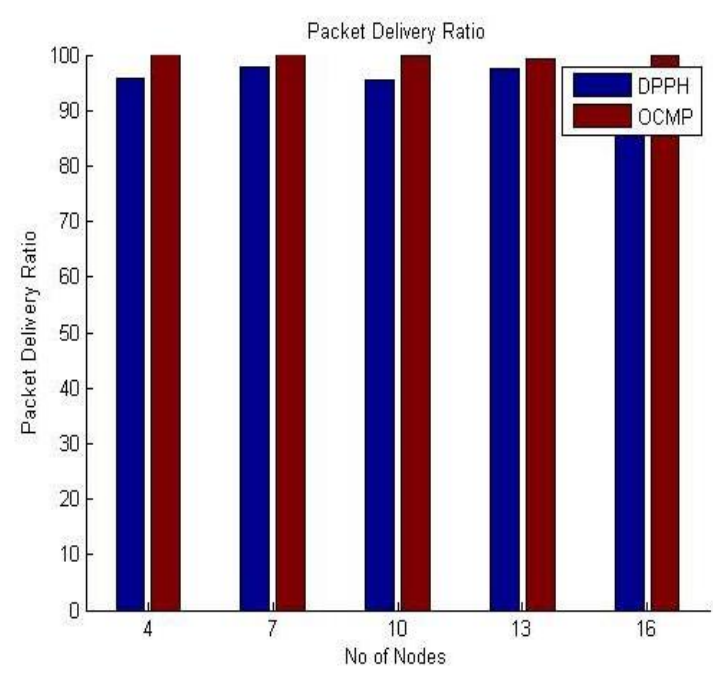

Fig.2. The Comparison graph for PDR.

\section{B. Throughput}

It is the number of packets/bytes received by the source per monitoring time. It is an important metric for analyzing network protocols. It calculates the total number of packets received at the receiver side with respect to the total monitoring time. The total throughput is estimated from Eq. 14.

$$
T H=\frac{\left(\sum_{k=1}^{n} P_{k}^{\text {Transmit }}-\sum_{k=1}^{n} P_{k}^{\text {Lost }}\right) *\left(P_{\text {Size }}\right)}{T_{\text {MonitorTimeStop }} T_{\text {MonitorTimeStart }}}
$$

where $P^{\text {Lost }}$ denotes the number of packets lost in the transmission, $P^{\text {Transmit }}$ denotes the number of packets transmitted from the source node, $P_{\text {Size }}$ denotes the size of a packet, $T_{\text {MonitorTimestop }}$ denotes the time when The patient monitoring is stopped, and $T_{\text {MonitolTimestart }}$ denotes the time when the monitoring is started.

The comparison graph of throughput given in Fig. 3, displays that OCMP shows major variations for different node values. However, DPPH shows consistency results for variation of nodes. The DPPH has significant growth in throughput than OCMP. This is due to the proposed dynamic prioritization based classification unit.

\section{End-to-End Delay}

The delay is defined as the average time taken by a packet to arrive at the destination. It also includes the delay caused by route discovery process and the queuing time in data packet transmission. Only the data packets that successfully delivered to destinations are counted. The delay is calculated by subtracting packet sending time from the packet receiving time. 


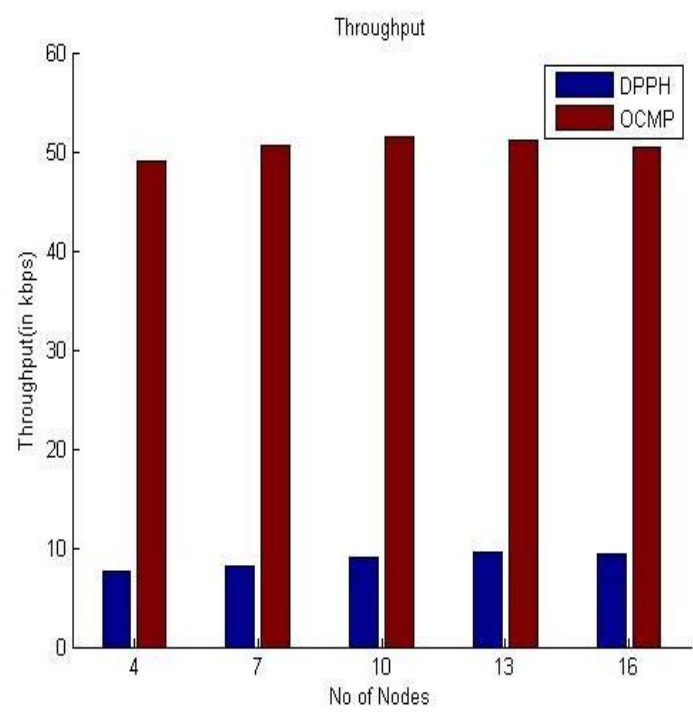

Fig.3. The Comparison graph for Throughput.

The total end to end is calculated from the equation given in Eq. (15).

$$
E 2 E_{\text {Delay }}=\frac{\sum_{k=1}^{n}\left(P_{k}^{\text {RcvTime }}-P_{k}^{\text {SndTime }}\right)}{\left.\sum_{k=1}^{n} P_{k}^{\text {Transmit }}-\sum_{k=1}^{n} P_{k}^{\text {Lost }}\right)} * 100
$$

where $P^{\text {Lost }}$ denotes the number of packets lost in the transmission, $P^{\text {Transmit }}$ denotes the number of packets transmitted from the source node, $P^{\text {RcvTime }}$ denotes the time in which the packet arrives at the destination, and $P^{\text {SndTime }}$ the time when a packet is sent from the source.

The simulation results obtained from Fig. 4 shows that the comparis on of delay for proposed protocol is nearly same with respect to a number of nodes.

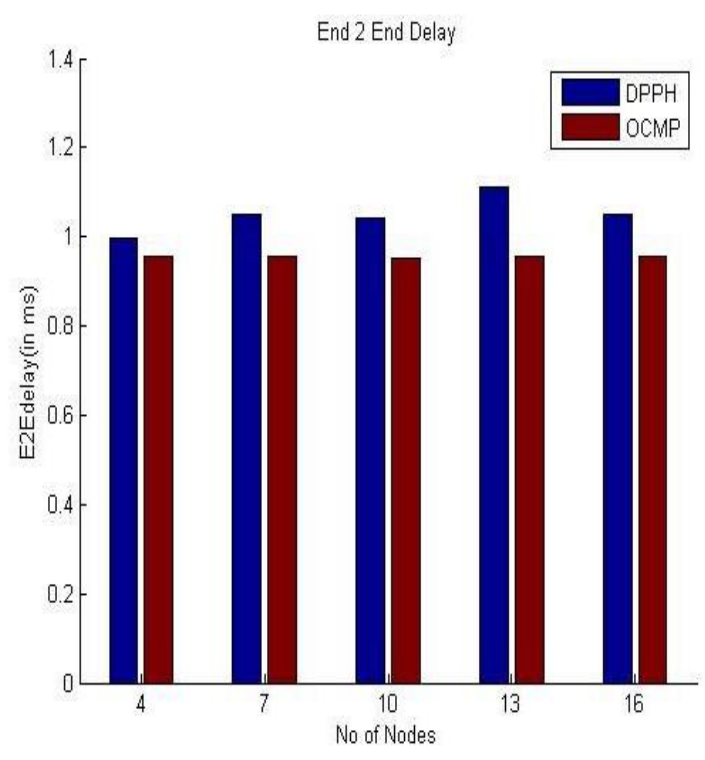

Fig.4. The Comparison graph for E2E Delay.

Also, the variation in delay in each case is negligible for both protocols, but delay occurring in DPPH is less as compared to OCMP protocol, which is a good significance for enhancement in network throughput.
The monitoring of a patient has been conducted for a time interval of 60 minutes. The patient was implanted with different types of sensor nodes. The number of sensor nodes is varied with time. The implementation was done through network simulator ns-2.35 for this monitoring time interval. The generated result shows that the proposed protocol significantly improves the network performance by increasing packet delivery ratio and throughput while reducing the delay.

\section{CONCLUSION}

A critical healthcare system is well designed for vital signal monitoring and determining the real-time conditions. Our proposed protocol implements a hybrid Binary Decision Tree and Support Vector Machine (BDSVMT) based classification mechanism, which ensures the delivery of critical and important packets in an urgent manner. This classifier is designed to provide superior multi-class classification by utilizing a BDT architecture that requires much less computation for deciding a class for unknown sample and SVM, that utilizes distance measures at the Kernel space to convert the multi-class problem into a binary decision tree. The dynamically updating priority helps for sensing and transmitting more important data within significant delay and minimum loss. The simulation results validated and analyzed that the proposed protocol improves the packet delivery ratio throughput in a better way with the reduction in packet transmission and queuing delay.

\section{REFERENCES}

[1] G. N. Bradai et al.,"QoS architecture over WBANs for remote vital signs monitoring applications", 12th Annual IEEE Consumer Communications and Networking Conference, pp. 1-6, 2015.

[2] M. Kathuria, and S. Gambhir, "Quality of service provisioning transport layer protocol for WBAN system", International Conference on Optimization, Reliability and Information Technology (IEEE Xplore), pp. 222-228, 2014.

[3] M. A. Ameen, A. Nessa, and K. S. Kwak, "QoS Issues with Focus on Wireless Body Area Networks", Third International Conference on Convergence and Hybrid Information Technology, vol. 1, pp. 801-807, 2008.

[4] M. Kathuria, and S. Gambhir, "Layer wise Issues of Wireless Body Area Network: A Review", International conference on Reliability, Infocom Technologies and Optimization (ICRITO), pp. 330-336, Jan 2013.

[5] S. Misra, V. Tiwari, and M. S. Obaidat," "LACAS: Learning Automata-Based Con gestion Avoidance Scheme for Healthcare Wireless Sensor Networks", IEEE Journal on Selected Areas in Communications, vol. 27, no.4, pp. 466-479, 2009.

[6] S Gambhir, V. Tickoo, and M. Kathuria, "Priority based congestion control in WBAN", Eighth International conference on contemporary computing (SCOPUS, DBLP, IEEE Xplore), pp. 428-433, 2015.

[7] N. Farzaneh and M. H. Yaghmaee, "Joint Active Queue Management and Congestion Control Protocol for Healthcare Applications in Wireless Body Sensor Networks", 9th International Conference on Smart Homes 
and Health Telematics (Springer Verlag), pp. 88-95, 2011.

[8] N. Farzaneh, M. H. Yaghmaee, and D. Adjeroh, "An Adaptive Congestion Alleviating Protocol for Healthcare Applications in Wireless Body Sensor Networks: Learning Automata Approach", Journal of Science and Technology (Springer), vol. 44, no. 1, pp. 31-4 1, 2012.

[9] M. H. Yaghmaee, N. F. Bahalgardi, and D. Adjeroh, "A Prioritization Based Congestion Control Protocol for Healthcare Monitoring Application in Wireless Sensor Networks", Wireless Personal Communications (Springer), vol. 72, no. 4, pp. 2605-2631, April 2013.

[10] A. A. Rezaee, M. H. Yaghmaee, and A. M. Rahmani, "Optimized Congestion Management Protocol for Healthcare Wireless Sensor Networks”, Wireless Personal Communications (Springer), vol. 75, no. 1, pp. 11-34, 2013.

[11] M. Kathuria, and S. Gambhir, "Reliable delay sensitive loss recovery protocol for critical health data transmission system", 2015 International Conference on Futuristic Trends on Computational Analysis and Knowledge Management (IEEE Xplore), pp.333-339, Feb 2015.

[12] Y. Liu et al.,'Hard and Soft Classification? Large margin unified machines", Taylor and Francis, vol. 106, pp. 166177, 2011.

[13] D. E.Taylor, and J. S. Turner, "ClassBench: a packet classification benchmark", IEEE/ACM Transactions on Networking, vol. 15, no. 3, pp. 499-511, 2007.

[14] D. E. Taylor, "Survey and taxonomy of packet classification techniques", ACM Computing Surveys (CSUR), vol. 37, no. 3, pp. 238-275, 2005.

[15] F. Huang, and Y. Lu-Ming, "Research on classification of hyper spectral remote sensing imagery based on BDTSMO and combined features," Journal of Multimedia, vol. 9, no. 3, pp. 456-462, 2014.

[16] N. Xue, "Comparison of multi-class support vector machines", Computer Engineering and Design, vol. 32, no. 5, pp. 1792-1795, 2011

[17] Q. Ai, Y. Qin, and J. Zhao, "An improved directed acyclic graphs support vector machine", Computer Engineering and Science, vol. 33, no. 10, pp. 145-148, 2011.

[18] G. Feng, "Parameter optimizing for Support Vector Machines classification", Computer Engineering and Applications, vol. 47, no. 3, pp. 123-124, 2011.

[19] C. C. Chung, and C. J. Lin, "LIBSVM: A library for support vector machine", ACM Transactions on Intelligent System and Technology, vol. 2, pp.1-27, 2011.

[20] M. Kathuria, and S. Gambhir, "Leveraging machine learning for optimize predictive classification and scheduling E-Health traffic", International Conference on Recent Advances and Innovations in Engineering (IEEE Xplore), p p . 1-7, 2014.

[21] L. Wenlong, and X. Changzheng, "Parallel Decision Tree Algorithm Based on Combination", IEEE International Forum on Information Technology and Applications (IFITA) Kunming, pp.99-101, July 2010.

[22] M. Kathuria, and S. Gambhir, "Genetic Binary Decision Tree based Packet Handling schema for WBAN system", Recent Advances in Engineering and Computational Sciences (IEEE Xplore). pp. 1-6. 2014.

[23] S. Geetha, N. Ishwarya, and N. Kamaraj, "Evolving decision tree rule based system for audio stego anomalies detection based on Hausdorff distance statistics", Information Sciences Journal (Elsevier Publisher), vol. 180, no. 13, pp. 2540-2559, 2010.

[24] K. Bhaduri, R. Wolff, C. Giannella, and H. Kargupta, "Distributed Decision-Tree Induction in Peer-to-Peer
Systems", Journal Statistical Analysis and Data Mining (John Wiley and Sons), vol. 1, pp. 1-35, June 2008.

[25] D. Kocev, C. Vens, J. Struyf, and S. Dzeroski, "Ensembles of multi-objective decision trees", 18th European Conference on Machine Learning (DBLP,Springer), pp.624-631, 2007.

[26] G. Madzarov, D. Gjorgjevikj, and I. Chorbev, "A Multiclass SVM Classifier Utilizing Binary Decision Tree", Informatics, vol. 33, pp.233-241, 2009.

[27] X. Wang and Y. Qin, "Research on SVM multi-class classification based on binary tree, "Journal of Hunan Institute of Engineering, vol. 18, pp. 68-70, 2008.

[28] G. Madzarov, D. Gjorgjevikj, and I. Chorbev, "Multiclass classification using support vector machines in decision tree architecture", IEEE EUROCON 2009 (EUROCON '09), pp. 288-295, 2009.

[29] K. K. Reddy, and V. Reddy, "A Survey on Issues of Decision Tree and Non-Decision Tree Algorithms", International Journal of Artificial Intelligence and Applications for Smart Devices(SERSC), vol. no. 1, pp. 932, 2016.

[30] S. Gambhir and M. Kathuria, "DWBAN: Dynamic Priority based WBAN Architecture for Healthcare System", 3rd International Conference on Computing for Sustainable Global Development (IEEE Xplore), pp 3380-3386, 2016.

[31] M. Kathuria, and S. Gambhir, " Comparion Analysis of proposed DPPH protocol for Wireless Body Area Network", International Journal of Coputer Applications (IJCA), vol. 144, pp. 36-41, 2016.

[32] M. Kathuria, and S. Gambhir, "Security and Privacy Assault of Wireless Body Area Network System", International conference on Reliability, Infocom Technologies and Optimization (ICRITO), pp: 223-229, Jan 2013

\section{Authors' Profiles}

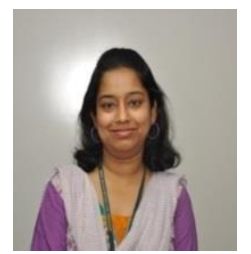

Madhumita Kathuria is Assistant Professors in Computer Science and Engineering Department Manav Rachna International University, Faridabad, India. She is pursuing her $\mathrm{PhD}$ (Computer Science \& En gineering) from YMCA University of Science and Technology. She has published more than 15 papers in various International and National journals and conferences. Her area of interests includes Wireless Body Area Network, Sensor Network, Network Security, Digital Image Processing, Learning and Computational Techniques.

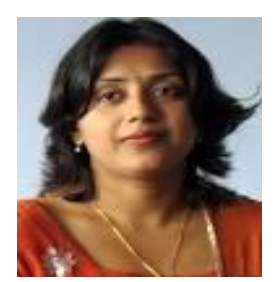

Dr. Sapna Gambhir is Assistant Professor in Computer Engineering Department in YMCA University of Science and Technology, Faridabad, India. She has done her PhD from Jamia Millia Islamia University in 2010. She has published more than 50 papers in various National and International journals and conferences. Her area of interests includes Wireless Sensor Network, Ad-hoc Network and Social Network, and Security of Wireless Networks. 
How to cite this paper: Madhumita Kathuria, Sapna Gambhir, "Performance Optimization in WBAN Using Hybrid BDT and SVM Classifier", International Journal of Information Technology and Computer Science(IJITCS), Vol.8, No.12, pp.83-90, 2016. DOI: $10.5815 /$ ijitcs.2016.12.10 